\title{
Multimedia information retrieval: best papers and expanding frontiers
}

\author{
Michael S. Lew
}

Published online: 9 April 2014

(C) Springer-Verlag London 2014

On behalf of the editorial board, it is an honor to present extended versions of the best conference papers from the field of multimedia research. The papers were selected initially based on the reviews of the program committees of well-known multimedia retrieval meetings (special thanks to the program chairs-ICMR: Marcel Worring, John Smith and Tat-Seng Chua; CBMI: Klaus Schoffmann and Tamas Sziranyi) and passed through another round of reviews by the IJMIR editorial board. From the research in 2013, we present two outstanding papers: "Context Assisted Face Clustering Framework with Human-in-the-Loop" and "Parallel Incremental Power Mean SVM for the Classification of Large Scale Image Datasets".

Grouping images of the same person together or face clustering is an important part of face tagging and recognition. In the prior research, there had been numerous systems which could achieve high precision but at the cost of high recall. The paper "Context Assisted Face Clustering Framework with Human-in-the-Loop" by Liyan Zhang, Dmitri V. Kalashnikov and Sharad Mehrotra presents a promising approach which leverages context information to learn adaptive rules for automatic and human-in-the-loop face clustering.

Very large-scale visual concept detection with millions or billions of images requires dealing with challenges in time and space complexity as well as imbalanced data. The paper

M. S. Lew $(\varangle)$

Leiden, The Netherlands

e-mail: mlew@liacs.nl
"Parallel Incremental Power Mean SVM for the Classification of Large Scale Image Datasets" by Thanh-Nghi Doan, Thanh-Nghi Do and Francois Poulet introduces extensions to Power Mean SVM to address the challenges mentioned above and gives promising results when working with 1,000 visual classes.

In addition, this issue has two interesting regular papers. The paper, "The Video Browser Showdown: A Live Evaluation of Interactive Video Search Tools" by Klaus Schoeffmann, David Ahlstrom, Werner Bailer, Claudiu Cobarzan, Frank Hopfgartner, Kevin McGuinness, Cathal Gurrin, Christian Frisson, Duy-Dinh Le, Manfred Del Fabro, Hongliang Bai and Wolfgang Weiss presents an overview and results gained from a video search competition. Useful insights are also given regarding the usage of sophisticated vs. simple tools in interactive video exploration.

One of the next frontiers of web search integrates user sentiment effectively into the search process. The paper "Information Extraction from Multimedia Web Documents: An Open-Source Platform and Testbed" by David Dupplaw, Michael Matthews, Richard Johansson, Giulia Boato, Andrea Costanzo, Marco Fontani, Enrico Minack, Elena Demidova, Roi Blanco, Thomas Griffiths, Paul Lewis, Jonathon Hare and Alessandro Moschitti describes a state-of-the-art system which utilizes opinion and sentiment analysis as well as pictorial features in the web search process.

Our research community is constantly expanding the frontiers in searching and exploring multimedia. Just in the past decade, we have seen the bar rise from recognizing 10 visual concepts to over 1,000. Moreover, we have seen multimedia search become a fundamental pillar of the major web search engines and an important tool in the advancement of the natural sciences. My thanks go out to all of the researchers and practitioners who comprise the multimedia retrieval community. 
Michael S. Lew

Editor-in-Chief

International Journal of Multimedia Information Retrieval

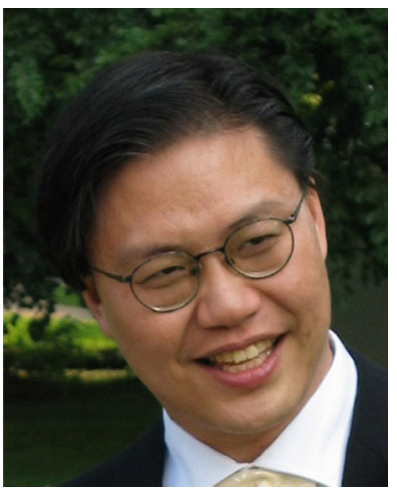

